\title{
An Unusual Case of Concurrent Central Retinal Vein and Cilioretinal Artery Occlusion in a Healthy Patient
}

\author{
Eunice Jin Hui Goh ${ }^{a}$ Kong Yong Goh ${ }^{b}$ \\ aMinistry of Health Holdings, Singapore, Singapore; bMount Elizabeth Novena Hospital, \\ Singapore, Singapore
}

\section{Keywords}

Hyperhomocysteinemia - Central retinal vein occlusion · Cilioretinal artery occlusion .

Methylenetetrahydrofolate reductase

\begin{abstract}
It is rare for young, healthy patients to have retinal venous or arterial occlusions and even rarer for both to occur in concert. Such an occurrence should prompt a rapid and extensive workup to prevent further complications. We present our patient, a 37-year-old Lebanese male, who reported a 3-day history of blurring of vision in his left eye. He had no medical or ocular history and is a nonsmoker. Examination of the left fundus revealed inferior macular edema and retinal whitening associated with tortuous retinal veins. He was diagnosed with a combined central retinal vein and cilioretinal artery occlusion. Emergency treatment was done for an acute arterial occlusion. Embolic and thrombotic causes were excluded with investigations. The only positive result was homozygosity for $677 \mathrm{C}>\mathrm{T}$ mutation of the 5,10 methylenetetrahydrofolate reductase (MTHFR) enzyme gene. MTHFR enzyme breaks down homocysteine, which is atherogenic and prothrombotic. This mutation can lead to a prothrombotic state, precipitating this occurrence. In fact, the Lebanese population is known to have the highest incidence of such mutations, but there are surprisingly few reports on retinal vascular occlusions attributed to this. He was promptly treated with antiplatelet therapy, possibly preventing a full-blown central retinal vein occlusion. After 4 weeks, his vision improved to $6 / 6$ bilaterally. Examination showed less tortuous veins, no more retinal whitening, resolution of macula edema and visual field defect. Hyperhomocysteinemia can be significant in patients without ischemic risk factors. It is vital to manage these patients promptly, preventing future sight and life-threatening events.
\end{abstract}




\section{Introduction}

The rare occurrence of combined central retinal vein occlusion (CRVO) and cilioretinal artery occlusion (CLAO) was first described by Oosterhuis [1]. Despite being previously reported, its pathogenesis remains to be subject to question. This case highlights the importance of suspecting an underlying condition in such combined occlusions as it could play a part in avoiding sight-threatening complications.

\section{Case Report/Case Presentation}

A 37-year-old healthy Lebanese male presented with a new, acute onset of blurring of vision for 3 days in his left eye (OS). On examination, his visual acuity was $6 / 6-1$ in the right eye (OD) and 6/9 + 2 in the left. His intraocular pressures and color vision were normal. Anterior segment examination of both eyes was unremarkable. There was no relative afferent pupillary defect. Fundal examination of the left eye revealed retina whitening along with the distribution of the cilioretinal artery, with inferior macula involvement. No emboli were seen. The retinal veins were also noted to be mildly tortuous and dilated (shown in Fig. 1). His right eye fundal examination was otherwise normal.

Further history was elicited to rule out cardiovascular risk factors. He was a nonsmoker, with no previous cerebrovascular events, myocardial infarction, pulmonary embolism, hypertension, or hyperlipidemia. He was not on any medications (including traditional). He also had no family history of thromboembolic events.

Investigations showed OS subfoveal retinal thickening and edema on optical coherence tomography (shown in Fig. 2) and a left super-central defect on automated perimetry. Fundus fluorescein angiography demonstrated that the cilioretinal artery had incomplete filling. The retinal veins were also noted to be tortuous.

He was suspected to have a CLAO and an impending CRVO. In this case, given his good vision, it was likely that the CLAO had spared the fovea.

Systemic examination of the patient was unremarkable. He had normal blood pressure and heart rate. On auscultation, there was no carotid bruit or cardiac murmur heard. In view of the patient's young age and lack of ischemic risk factors, his ocular presentation prompted a search for an embolic, thrombotic, or vasculitic cause to explain the phenomena.

Cardiac investigations were unremarkable. His electrocardiogram was in sinus rhythm with a normal QRS axis, QT interval, and no significant ST or T wave changes. Echocardiography demonstrated preserved left ventricular size and function (ejection fraction 65-70\%), normal

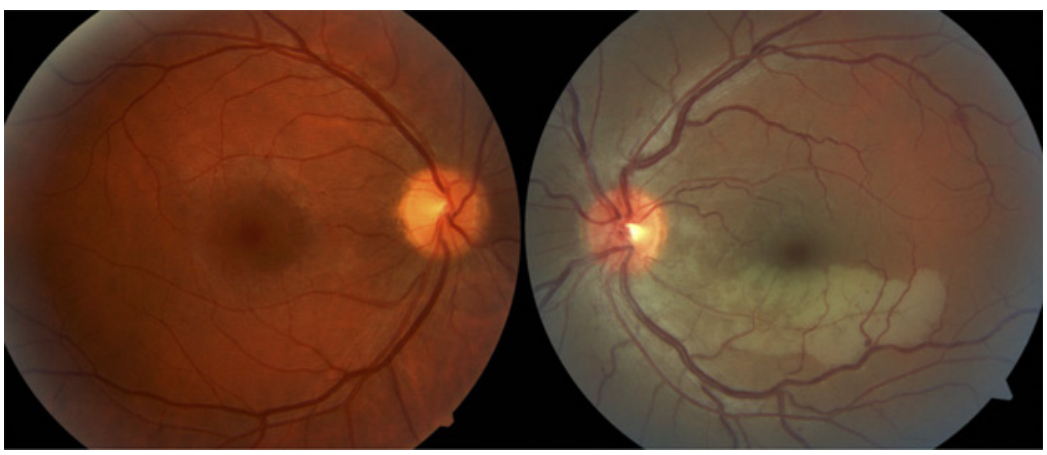

Fig. 1. OD, normal fundal examination; OS, retinal whitening along with the distribution of the cilioretinal artery (arrow) with mildly tortuous and dilated retinal veins. OD, left eye; OS, right eye. 


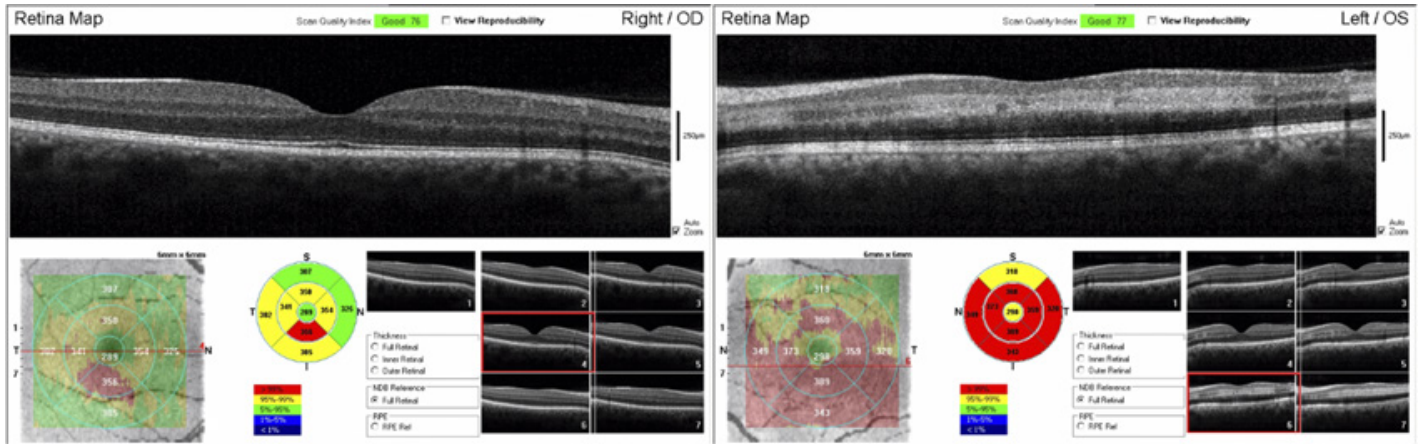

Fig. 2. OCT showed OS subfoveal retinal thickening and edema. OCT, optical coherence tomography; OS, right eye.

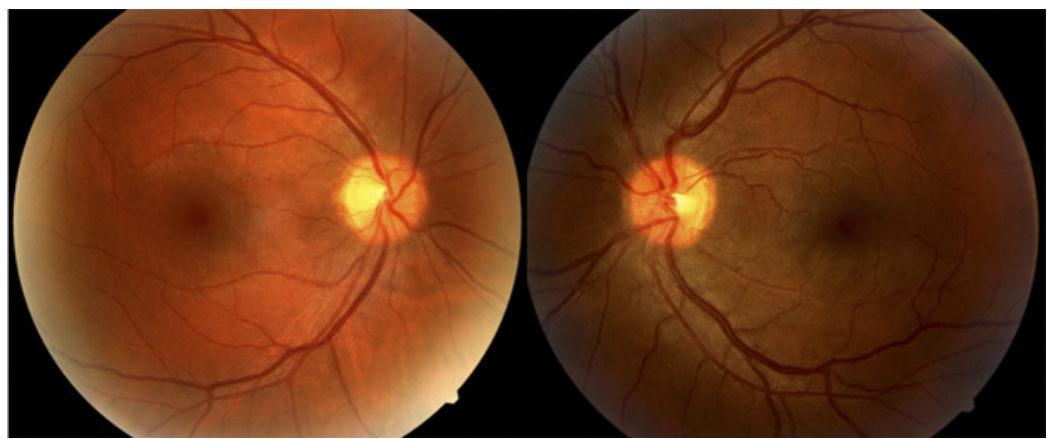

Fig. 3. OS less tortuous retinal veins and resolution of retinal whitening. OS, right eye.

atrial sizes, mild tricuspid regurgitation, no patent foramen ovale or interatrial communication, and no clot in his left atrial appendage. Carotid duplex showed no carotid plaque or stenosis. Last, he had a 5-days Holter cardiac monitoring done which showed sinus rhythm throughout, isolated ventricular ectopics with no arrhythmias, or atrial fibrillation detected.

Laboratory tests were done to rule out thrombotic and vasculitic causes. It revealed that the patient was $677 \mathrm{C}$-T mutation (homozygous positive) for the 5,10 methylenetetrahydrofolate reductase (MTHFR) gene. He also had a suboptimally high level of homocysteine (10.24 $\mu \mathrm{mol} / \mathrm{L})$. Other extensive systemic workup including 25-hydroxycholecalciferol, anti-b2GP1, anticardiolipin IgG/M, anti-MPO, anti-PR3, antithrombin III, C-reactive protein, factor V Leiden, ferritin, homocysteine, lupus anticoagulant, protein $\mathrm{C}$ activity, protein S activity, prothrombin gene polymorphism, and treponema pallidum antibody were normal. He was treated prophylactically with lifelong antiplatelet therapy (aspirin) with consideration of full anticoagulation should another thrombotic episode occur.

After 4 weeks, his vision was $6 / 6$ bilaterally. The retinal veins appeared to be less tortuous and retinal whitening had resolved (shown in Fig. 3). It was noted that his macular edema and visual field defect had resolved.

\section{Discussion}

It is rare for young, healthy patients to have a venous or arterial occlusion and even rarer for both to occur in concert. Mechanisms causing arterial occlusions include embolism, thrombosis, vasospasm, and vasculitis. On the other hand, venous occlusions are caused by thrombosis, compression, and vasculitis. 
In our patient, we suggest a 677C-T mutation (homozygous positive) of the MTHFR enzyme gene polymorphism as a possible inciting factor. The MTHFR enzyme is important in the breakdown of the body's homocysteine by reducing 5,10-methylenetetrahydrofolate to $\mathrm{N}$-5-methyltetrahydrofolate (MTHF) in the folate cycle [2, 3]. A 677C-T mutation involves a change in the amino or splice site of alanine to valine at exon 4 . Such gene polymorphisms can lead to moderate to severe hyperhomocysteinemia [4].

Homocysteine, a sulfur-containing amino acid, has been found to be associated with premature atherosclerosis, coronary artery disease, stroke, and peripheral arterial disease [5]. An elevated total homocysteine level has been established as a risk factor for arterial and/ or venous thrombosis by inducing endothelial dysfunction through oxidative stress, triggering oxidation of low-density lipoprotein, stimulating smooth muscle cell proliferation and prothrombotic state and, hence, causing possible combined retinal vein and arterial occlusion [6].

It has been reported that the Lebanese population has the highest incidence of MTHFR A1298C polymorphisms (another known mutation of MTHFR) among previously reported populations such as the Indians and Chinese who had the lowest frequency [7, 8]. Although bearers of the MTHFR A1298C polymorphisms do not exhibit hyperhomocysteinemia, compound heterozygotes for $677 \mathrm{C}-\mathrm{T}$, like our patient, may be at risk for hyperhomocysteinemia and low folate levels. Despite the high prevalence of MTHFR polymorphisms in Lebanon, it is interesting to note that there exist little reports on patients with retinal vascular occlusions attributed to hyperhomocysteinemia, as compared to other causes such as antiphospholipid and anticardiolipin antibodies. This could suggest that there may be Lebanese patients deemed to have "idiopathic" retinal vascular occlusions whom might have undiagnosed hyperhomocysteinemia that is amenable to treatment.

Based on previously published literature, it is postulated that CRVO precedes CLAO. This is related to the perfusion pressure in the cilioretinal artery and resistance to perfusion created by CRVO. When the central retinal vein is occluded, it results in an elevation of intraluminal capillary pressure (central retinal artery continues perfusing retina). However, the cilioretinal artery, being supplied by the posterior ciliary artery circulation, has a lower perfusion pressure than the central retinal artery. This results in a relative occlusion of the cilioretinal artery $[9,10]$.

There is currently no consensus in terms of treatment of hyperhomocysteinemia. The Norwegian Vitamin Trial (2006) investigated the lowering of homocysteine levels with folic acid and vitamin B12 for secondary prevention of cardiovascular disease after an acute myocardial infarction. Although a $27 \%$ reduction of plasma homocysteine levels was achieved, there was no associated risk reduction for myocardial infarction or stroke [11,12].

Given the current evidence, it would be best to manage additional risk factors for atherosclerosis or venous thrombosis conservatively. In our case, we started him on aspirin for primary prevention for possible future similar events. Rapid initiation of aspirin could have averted the occurrence of a full-blown central retinal vein occlusion, which might have resulted in undesirable complications like cystoid macular edema and neovascularization glaucoma.

\section{Conclusion}

Our case highlights the significance of hyperhomocysteinemia in patients with minimal ischemic risk factors presenting with concurrent retinal arterial and venous occlusions. In a Lebanese patient, it would be all the more important to consider this as a cause simply based on its high prevalence of MTHFR polymorphisms. It is vital to investigate the cause and 
Goh and Goh: Concurrent Retinal Vein and Artery Occlusion in a Healthy Patient

manage these patients appropriately to prevent future recurrences of vascular events like a central retinal artery occlusion which can be sight-threatening.

\section{Statement of Ethics}

Written informed consent was obtained from the patient for publication of this case report and accompanying images. A copy of the written consent is available for review upon request.

\section{Conflict of Interest Statement}

The authors have no conflicts of interest to declare.

\section{Funding Sources}

The authors have no funding sources to declare.

\section{Author Contributions}

Eunice Jin Hui Goh was involved in writing - original draft, writing - review and editing, and visualization of this case report. Kong Yong Goh was involved in conceptualization and supervision of this report.

\section{References}

1 Oosterhuis JA. Fluorescein fundus angiography in retinal vein occlusion. In: Henkes HE, editor. Perspectives in ophthalmology. Amsterdam: Excerpta Medica Foundation; 1968:29-47.

2 Frosst P, Blom HJ, Milos R, Goyette P, Sheppard CA, Matthews RG, et al. A candidate genetic risk factor for vascular disease: a common mutation in methylenetetrahydrofolate reductase. Nat Genet. 1995;10(1):111-3.

3 McGimpsey SJ, Woodside JV, Bamford L, Gilchrist SE, Graydon R, McKeeman GC, et al. Retinal vein occlusion, homocysteine, and methylene tetrahydrofolate reductase genotype. Invest Ophthalmol Vis Sci. 2005;46(12): 4712-6.

4 Eldibany MM, Caprini JA. Hyperhomocysteinemia and thrombosis: an overview. Arch Pathol Lab Med. 2007; 131(6):872-84.

5 Fekih-Mrissa N, Mrad M, Klai S, Mansour M, Nsiri B, Gritli N, et al. Methylenetetrahydrofolate reductase (C677T and A1298C) polymorphisms, hyperhomocysteinemia, and ischemic stroke in Tunisian patients. J Stroke Cerebrovasc Dis. 2013;22(4):465-9.

6 Ghaznavi H, Soheili Z, Samiei S, Soltanpour MS. Plasma homocysteine levels, methylene tetrahydrofolate reductase A1298C gene polymorphism and risk of retinal vein thrombosis. Blood Coagul Fibrinolysis. 2016; 27(6):679-83.

7 Sabbagh AS, Mahfoud Z, Taher A, Zaatari G, Daher R, Mahfouz RA. High prevalence of MTHFR gene A1298C polymorphism in Lebanon. Genet Test. 2008;12(1):75-80.

8 Almawi WY, Finan RR, Tamim H, Daccache JL, Irani-Hakime N. Differences in the frequency of the C677T mutation in the methylenetetrahydrofolate reductase (MTHFR) gene among the Lebanese population. Am J Hematol. 2004;76(1):85-7.

9 Schatz H, Fong AC, McDonald HR, Johnson RN, Joffe L, Wilkinson CP, et al. Cilioretinal artery occlusion in young adults with central retinal vein occlusion. Ophthalmology. 1991;98(5):594-601.

10 Keyser BJ, Duker JS, Brown GC, Sergott RC, Bosley TM. Combined central retinal vein occlusion and cilioretinal artery occlusion associated with prolonged retinal arterial filling. Am J Ophthalmol. 1994;117(3):308-13.

11 Maron BA, Loscalzo J. The treatment of hyperhomocysteinemia. Annu Rev Med. 2009;60:39-54.

12 Bonaa KH, Njolstad I, Ueland PM, Schirmer H, Tverdal A, Steigen T, et al. Homocysteine lowering and cardiovascular events after acute myocardial infarction. N Engl J Med. 2006;354(15):1578-88. 